\title{
Traces Detection Of Coronavirus Patients
}

\author{
Jigyasu Prakash¹, Kalyani Joshi², Manali Bhavsar ${ }^{3}$, \\ Suyash Sinha ${ }^{4}$, Yash Sharangpani ${ }^{5}$ and Shailendra Aote ${ }^{6}$ \\ ${ }_{1,2,3,4,5,6}$ Computer Science Department, Shri Ramdeobaba College of \\ Engineering and Management, Nagpur, Maharashtra, India.
}

\section{ABSTRACT}

COVID-19 is a disease caused by a new strain of coronavirus. With the rapid increase in the spread of COVID-19, many people are getting affected by it. As the disease cannot be detected until the affected person doesn't take the COVID-19 test, the person remains unaware that he has been infected by the coronavirus. The person would travel, go to shops, do other activities as well thereby infecting the other people and a potential threat to society. Thus it becomes difficult to trace all the people who have been infected. It is necessary to report all the close contacts of the infected person in the last 14 days. With the increase in the COVID-19 cases, it is very difficult to manually monitor and track down all the contacts of a COVID-19 positive patient. This calls for an autonomous application that will provide information about the person's traces, the people with whom he came in contact with and the places he visited in the last 14 days. This application will help in collecting the data for traces of a COVID-19 positive patient.

KEY WORDS: FACE API JS, COVID-19, FACE RECOGNITION, AADHAAR BASED FACE RECOGNITION, INTERNET OF THINGS (IOT),MERN.

\section{INTRODUCTION}

COVID-19 is an infectious disease caused by severe respiratory syndrome (SARS-CoV-2). This disease mainly spread between the people of proximity. It spreads via contaminated droplets during breathing, coughing, sneezing, and talking. COVID-19 is spreading in the world at alarming rates and is difficult to keep the records of the traces manually. To date, there is no specific medicine for treating this disease. The best way to prevent illness is to avoid being exposed to the virus. The system for detecting the traces of COVID-19 positive patients is developed to control the spread of the disease. It consists of five modules - detecting the person from the video

\section{ARTICLE INFORMATION}

Received 11th Oct 2020 Accepted after revision 24th Dec 2020 Print ISSN: 0974-6455 Online ISSN: 2321-4007 CODEN: BBRCBA

Thomson Reuters ISI Web of Science Clarivate Analytics USA and Crossref Indexed Journal

\section{Clarivate
Analytics}

NAAS Journal Score 2020 (4.31)

A Society of Science and Nature Publication,

Bhopal India 2020. All rights reserved.

Online Contents Available at: http//www.bbrc.in/

Doi: $h$ ttp://dx.doi.org/10.21786/bbrc/13.14/83 footage, recognizing that person, making a trace entry in the database, obtaining the traces with the timeline, and visualization of traces.

The hardware requirement for this application is a camera with at least resolution of $720 \mathrm{x} 480$ pixels, it can either be a mobile camera or CCTV camera deployed at public places like shops to capture the data on which the application will run. The application uses the face recognition algorithm for recognizing the face of the person who visits the shop. The trace of the person is marked against the Aadhaar card number of that person along with the current time and date in the database. The Aadhaar card database is used for face recognition. If the person who visited the shop tests COVID positive after some days then the last 15 days traces of the person can be obtained by entering the Aadhaar card number of the person in the web browser. The system for detecting the traces of COVID positive patients will be useful to take preventive measures at the places where the infected person has visited in the last 15 days. This will help to control the pace of spreading of the disease and will decrease the count of the infected people. 


\section{System Overview}

A) Detecting the person:

Video footage is captured by the camera.

B) Recognizing the person:

Implemented using Face API JS which is internally based on Tensorflow.

C) Obtaining Aadhaar number and sending it to the central server: REST API is used to send the data to the Express server.

D) Obtaining traces:

Based on the Aadhaar number, the query is executed on MongoDB to fetch the traces.

E) Visualization of thetraces

Traces are visualized using the admin panel which is made using ReactJS.

Figure 1: Flowchart of the system which shows overall working

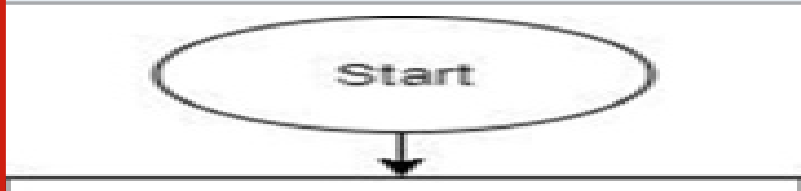

Person detected by camera
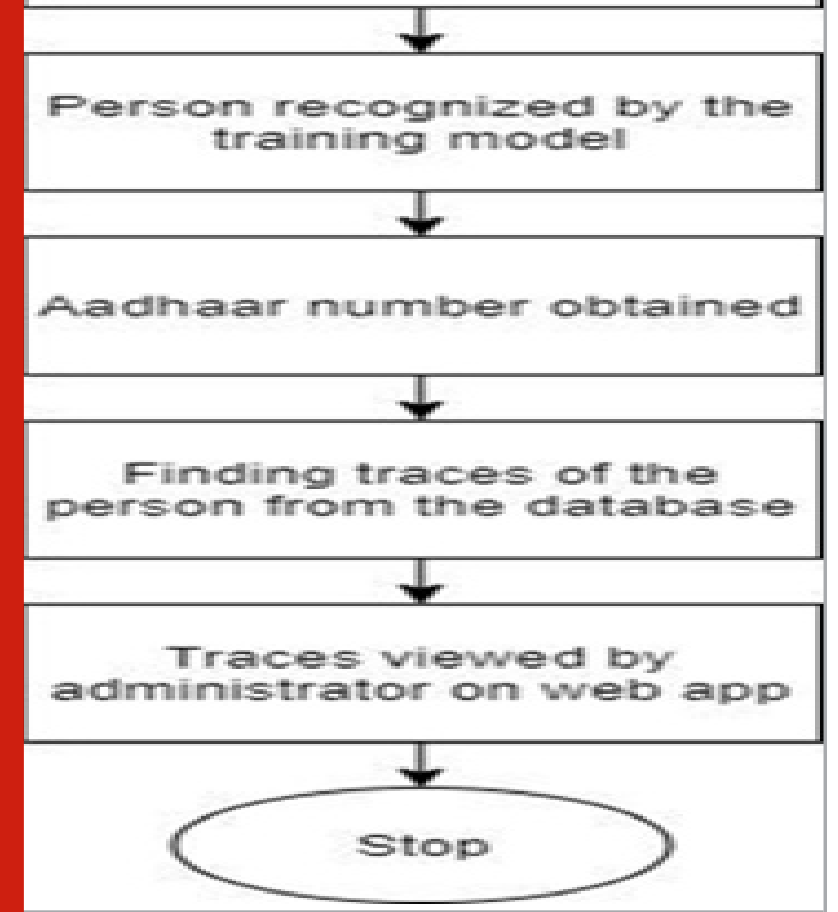

The language used in this project is JavaScript. It is a dynamic computer programming language. It is lightweight and most commonly used as a part of web pages, whose implementations allow client-side script to interact with the user and make dynamic pages. JavaScript can also be used in the backend by using the Node.js framework. The Node.js framework allows a developer to handle data updates from the front end and build scalable network applications that can process multiple simultaneous user requests. MongoDB is used as the database. It is an open-source document database and a leading NoSQL database. It is written in $\mathrm{C}++$. The data format of MongoDB is similar to JSON. MongoDB is highlyscalable.

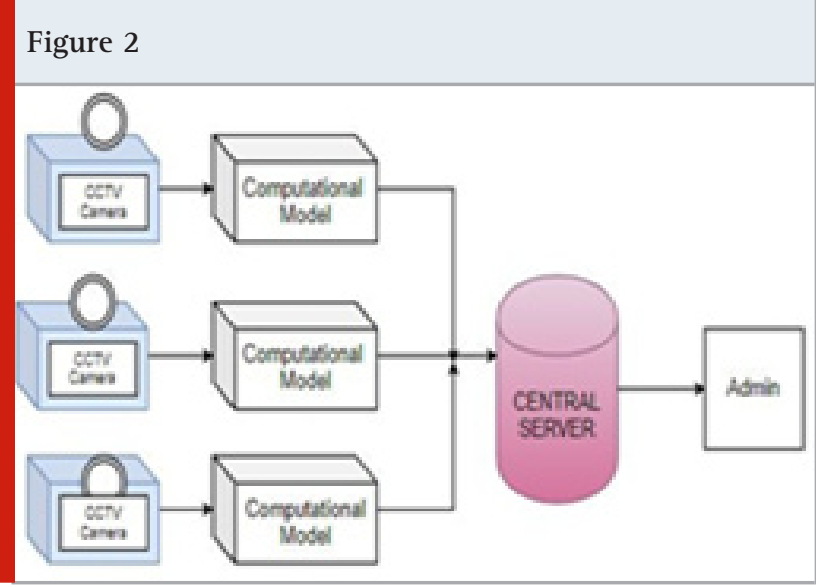

The key idea behind the working of the project is to store the traces of each person so that in the future if a person gets infected by COVID19. Authorities can find out the person's last 15 days trace easily and can take appropriate action to reduce the spread of COVID19.To achieve this goal whenever a person enters a shop or any public place. With the help of CCTV footage or the mobile phone of the shop owner, the person is detected and his Aadhaar card number is stored in the central server. In the future, if a person gets infected by COVID 19 his Aadhaar number will be entered on the website and the person's traces for the last 15 days will be showcased.

\section{System Implementation}

A. FaceRecognition: The first step is the implementation of a face recognition module to detect the person in the video footage of cameras that are placed at the entrance of different public places like shops, malls, etc. This module will recognize all the persons present in the video feed of the camera and independently will be accounted. A minified JSON object is collected at the server and will mark the trace entry in the database. The person will be recognized by the training model. For detecting faces successfully a Face API JS is implemented over Tensorflow JS core API.

Figure 3: Input and output image showing a bounded box around the person and the recognized name of the person.

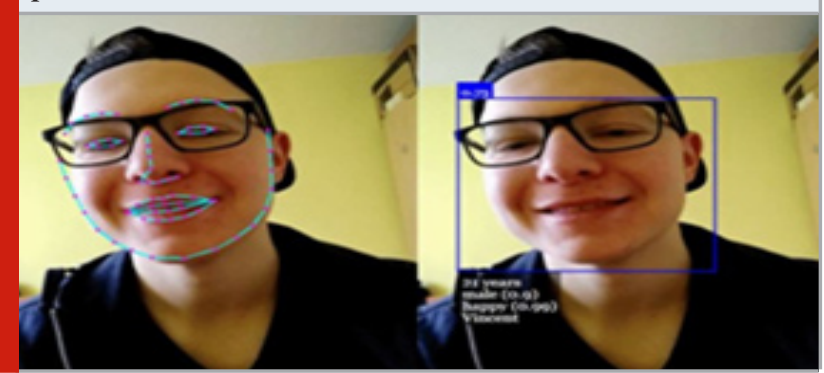


B. Obtaining the Aadhaarnumber: Fetching the Aadhaar number of the recognized person is a crucial step. The further processing is completely dependent on the Aadhaar number of the person. The Aadhaar number can be obtained by REST API which is used for fetching and updating data at the database end i.e. MongoDB using Express server. This data is further sent to the central server using the Express server.

C. Finding traces of theperson: With the help of the Aadhaar number, the details of the visits of that particular person in the last 15 days can be obtained. Based on the Aadhaar number, the query is executed on MongoDB using the REST API to fetch the traces of the person. The database also contains information about the date and time of the person's past visits to various shops which are also fetched.

D. Viewing Traces: The web administrator has the access to view the details of traces of a person and privileges to query Aadhaar details. The admin panel is designed by using React JS. The admin dashboard has a data field to enter the Aadhaar number of the person whose traces have to be obtained. An admin dashboard-Aadhaar query is executed and all the data present in the database against that Aadhaar number is displayed to admin. The details include the date, time, and places where the person has visited.

Figure 4: Dashboard displaying the traces of COVID-19 patient.

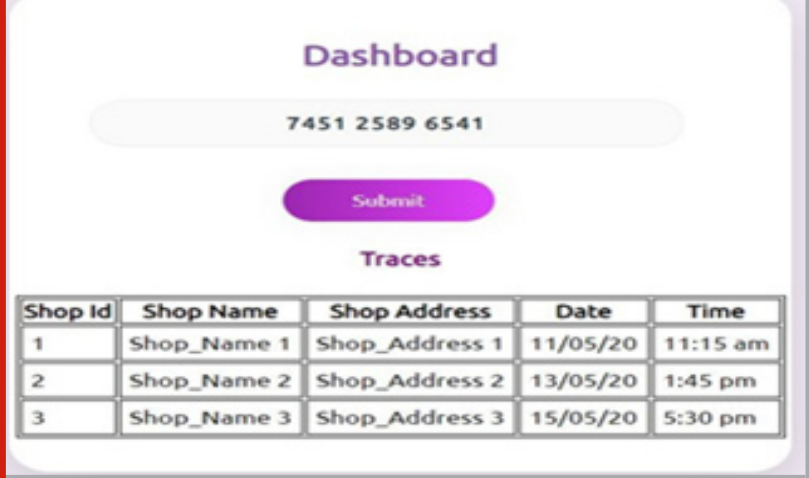

\section{RESULTS}

An algorithm is implemented to detect the past traces of the person if the person detects positive in the Corona Virus test. The system for detecting the traces of COVID positive patients will help to find the traces of the infected person and will help to analyze and control the spread at the places where the patients are increasing. This system helps to take preventive measures at the places where there are chances of the spread of the disease.

\section{CONCLUSION}

The system for detecting the traces of COVID-19 positive patients aims to control the spread of COVID-19. This system will complement the existing official government COVID-19 trackers and will be of great help to the government especially when the corona patient does not have access to the existing government COVID-19 trackers app. The main objective of this paper is to give in-depth information on the system for detecting the traces of COVID positive patients, the technology and the algorithm behind the whole working of the system, and how the whole system is beneficial for control of the spread of COVID and take preventive measures.

\section{REFERENCES}

Meena and R. Sharan, "An approach to face detection and recognition," 2016 International Conference on Recent Advances and Innovations in Engineering (ICRAIE), Jaipur, 2016, pp. 1-6, DOI: 10.1109/ ICRAIE.2016.7939462

Daniel Smilkov, Nikhil Thorat, Yannick Assogba, Ann Yuan, Nick Kreeger, Ping Yu, Kangyi Zhang,Shanqing Cai, Eric Nielsen, David Soergel, Stan Bileschi, Michael Terry, Charles Nicholson, Sandeep N. Gupta, Sarah Sirajuddin, D. Sculley, Rajat Monga, Greg Corrado, Fernanda B. Viégas, Martin Wattenberg," TensorFlow. js: Machine Learning for the Web and Beyond", SysML, Palo Alto, CA, USA (2019).

L.Yuan, Z. Qu, Y. Zhao, H. Zhang, andQ.Nian, ”A convolutional neural network based on TensorFlow for face recognition,” 2017 IEEE 2nd Advanced Information Technology, Electronic and Automation Control Conference (IAEAC), Chongqing, 2017, pp. 525-529, DOI:10.1109/IAEAC.2017.8054070.

Martin Abadi, Paul Barham, Jianmin Chen, Zhifeng Chen, Andy Davis, Jeffrey Dean, Matthieu Devin, Sanjay Ghemawat, Geoffrey Irving, Michael Isard, Manjunath Kudlur, Josh Levenberg, Rajat Monga, Sherry Moore, Derek G. Murray, Benoit Steiner, Paul Tucker, Vijay Vasudevan, Pete Warden, Martin Wicke, Yuan Yu, Xiaoqiang Zheng," TensorFlow: A system for largescale machine learning",12th USENIX Symposium on Operating Systems Design and Implementation (OSDI 16), USENIX Association (2016), pp.265-283 